\title{
pH-sensitive microemulsion-based gels for removal of colonic ammonia: A novel preventative oral preparation for hepatic encephalopathy in rats
}

\author{
CHUN-LE ZHANG ${ }^{1,5}$, ZHI-JUN DUAN ${ }^{1}$, GE TIAN ${ }^{2}$, YAN TIAN ${ }^{2}$, GAO-HONG HE ${ }^{3}$, \\ TENG-FEI BIAN ${ }^{3}$, XUE JIN $^{1}$, XIAO-YU SUN ${ }^{1}$, ZHEN LIU $^{1}$ and QING-YONG CHANG ${ }^{4}$ \\ ${ }^{1}$ Department of Gastroenterology, First Affiliated Hospital of Dalian Medical University, Dalian, Liaoning 116011; \\ ${ }^{2}$ Pharmaceutical College, Dalian Medical University, Dalian, Liaoning $116044 ;{ }^{3}$ Membrane Science and \\ Technology Center, Dalian University of Technology, Dalian, Liaoning 116012; ${ }^{4}$ Department of Neurosurgery, \\ Zhongshan Affiliated Hospital of Dalian University, Dalian, Liaoning 116001, P.R. China
}

Received January 23, 2014; Accepted September 19, 2014

DOI: $10.3892 / \mathrm{mmr} .2015 .3201$

\begin{abstract}
Microemulsions with limited stability in mimetic gastrointestinal environments have previously demonstrated potential for the effective removal of ammonia from artificial colonic fluid. Specialized pH-sensitive microemulsion-based gels for the removal of colonic ammonia (MBG-RCA), however, possess relative stability in the gastrointestinal (GI) tract of normal rats, indicating potential use in in vivo applications. An investigation of the effects of oral MBG-RCA was conducted in order to evaluate the reduction of intestinal ammonia and the prevention of hepatic encephalopathy (HE) in rat models. Eighty rats were allocated into eight 4-day treatment groups: The HE model (intraperitoneal injection of thioacetamide) group; the high-, medium- and low-dose MBG-RCA therapeutic groups $(15,10$ and $5 \mathrm{ml} / \mathrm{kg}$ MBG-RCA, respectively); and the normal, blank, lactulose and acetic acid control groups, each of which received daily treatment administration. Oral MBG-RCA effects were identified using behavioral monitoring observed by an infrared night vision supervisory control system, electroencephalograms, blood ammonia levels, intestinal ammonia levels, liver functionality and pathological
\end{abstract}

Correspondence to: Professor Zhi-Jun Duan, Department of Gastroenterology, First Affiliated Hospital of Dalian Medical University, 222 Zhongshan Road, Dalian, Liaoning 116011, P.R. China E-mail: cathydoctor@sina.com

Dr Qing-Yong Chang, Department of Neurosurgery, Zhongshan Affiliated Hospital of Dalian University, 6 Jiefang Street, Zhongshan, Dalian, Liaoning 116001, P.R. China

E-mail: qychang0409@163.com

Present address: ${ }^{5}$ First People's Hospital of Keerqin District in Tongliao, Tongliao, Inner Mongolia 028000, P.R. China

Key words: pH-sensitive gel, microemulsion-based gel, colonic ammonia removal, hepatic encephalopathy, preventative oral preparation observation. High- and medium-dose oral administrations of MBG-RCA significantly decreased the blood and intestinal ammonia levels $(\mathrm{P}<0.05)$, improved liver functionality and reduced the clinical manifestations of HE in rats. MBG-RCA demonstrated high clearance of rat colonic ammonia while maintaining sufficient stability in the GI tract, indicating the potential for the development of new clinically relevant oral preparations for the prevention of HE. Additionally, such preparations are advantageous in that ammonia is eliminated without the production of potentially harmful metabolic byproducts.

\section{Introduction}

Elimination of excess ammonia is the primary method of prevention and treatment of hepatic encephalopathy (HE), a progressive condition in which neurological function is impaired as the liver fails to remove toxic metabolites, particularly ammonia, from the bloodstream. The majority of intestinal ammonia is produced in the colon; however, no clinically viable oral treatment exists for the elimination of this excess ammonia, resulting in continuous low clearance levels due to poor ammonia elimination in the body.

$\mathrm{HE}$ is a syndrome caused by serious hepatic diseases as a result of metabolic disturbance and dysfunction of the central nervous system. Its clinical manifestation includes deposition changes, ethological abnormalities and mental retardation. In severe cases, it may develop into a coma or even be life-threatening. A number of researchers have divided HE into three categories: Type A is associated with acute liver failure; type B is caused by severe portosystemic shunting without liver disease; and type $\mathrm{C}$ is associated with chronic liver disease and hepatic cirrhosis (1). Acute liver failure models are well recognized as a representative model of type A HE. These models may be used to examine the pathological response of HE to various treatment methods in animal models (2).

HE may occur for a variety of reasons, and it is widely associated with numerous other pathological conditions. The classic theory holds that ammonia intoxication is the root cause of the 
symptoms, and a number of studies have demonstrated that the function of the cranial nerve is improved by the removal of intestinal ammonia (3-5). Ethological manifestations, electroencephalography (EEG), blood ammonia, intestinal ammonia, levels of alanine aminotransferase (ALT) and total bilirubin (TBIL) and liver pathology have each been used as previous indicators of HE in rat models and humans (6-8).

Effective treatment options for $\mathrm{HE}$ include reducing the bacterial production of ammonia and enhancing its elimination, inhibiting the growth of intestinal bacteria, regulating the amino acid metabolism and protecting brain function. Commonly used methods to counteract HE may be divided into several types according to treatment access: Administration via the oral, rectal or intravenous routes; artificial liver transplantation; and full liver transplantation (9-13). Oral drugs mainly act by reducing the production and absorption of ammonia. Drugs including lactulose, lactitol and kactitok operate by this mechanism $(14,15)$. These drugs pass through the small intestine without being absorbed or hydrolyzed and thus reduce the intestinal burden of ammonia by interacting with colonic bacteria to acidify the intestinal tract. This acidification increases the number of protein-synthesizing bacteria and reduces the number of protein-degrading bacteria $(16,17)$. These treatments, however, also have unpleasant side effects that include diarrhea and flatulence (18). Alternative medications, fradiomycin and rifaximin, decrease ammonia production by inhibiting bacterial RNA synthesis, thereby reducing intestinal ammonia as well as nervous and mental symptoms $(19,20)$. However, these treatments also have relatively serious renal and hepatic side effects.

Ammonia, considered to be the primary cause of $\mathrm{HE}$ symptoms, is principally produced in the colon by intestinal bacterial action from undigested protein and amino acids. A small amount of ammonia is generated by the diffusion of urea from the blood to the intestine, where it is hydrolyzed by bacterial urease, and an intestinal alkaline environment is conducive to the absorption of ammonia.

Microemulsions for the removal of colonic ammonia (ME-RCA) were prepared using a formulation containing Tween-80 (surfactant), ethylene glycol (cosurfactant), dimethyl silicone oil (external oil phase) and an ammonia absorbent with a solution of $30 \%$ acetic acid and $0.9 \%$ sodium chloride (internal phase). The principle was that acetic acid was used as the water phase for removing ammonia (an alkaline substance in accordance with the theory of acid-base balance). The surfactant and cosurfactant play a significant role in maintaining stability. Microemulsion has been widely used as a drug carrier from as early as the 1980s. Based on this observation, ammonia adsorbent acetic acid solution could be designed to be embedded in a microemulsion as an inner aqueous phase to establish ME-RCA (21). Previous studies have demonstrated that ME-RCAs are effective in the removal of ammonia in artificial colonic fluids; however, these compounds are not stable in the mimetic gastrointestinal (GI) environment, indicating the likelihood of poor effectiveness in vivo (21-24). These ME-RCAs could be further $\mathrm{pH}$-intelligentialized using macromolecular materials, including sodium alginate, to obtain pH-resistant MBG-RCAs suitable for oral administration.

MBG-RCA as a compound includes Tween-80, ethylene glycol, dimethyl silicone oil, acetic acid and sodium alginate.
The latter controls the release of drugs according to different $\mathrm{pH}$ values along the GI tract (25-27). In the acidic environment of the stomach, the sodium alginate gel contracts, without release of the drug, whereas it swells in certain $\mathrm{pH}$ environments ( $\mathrm{pH}$ range $7.4-10.4)(28,29)$ in the colon $(30,31)$. MBG-RCA reaches the colon following administration and swells to release ME-RCA. It absorbs intestinal ammonia through acid-base neutralization, and is then carried out of the body. Since the slightly acidic conditions of the colon, generally ranging from $\mathrm{pH}$ 5.5-7.0, are responsible for this instability, a $\mathrm{pH}$-sensitive microemulsion-based gel has been proposed for the removal of colonic ammonia (MBG-RCA). Macromolecular materials, including sodium alginate, enclose and protect the original ME-RCA, making it particularly resilient to the varied $\mathrm{pH}$ levels encountered upon oral administration. Furthermore, these molecules have been previously used as carriers to release bioactive molecules to targeted areas of the body, and this delivery system has been used to release ME-RCA directly in the colon (21). By adding a protective layer to existing microemulsion molecules, an increased resistance to $\mathrm{pH}$ may be achieved that increases the pharmacological usefulness of these materials in the treatment of HE.

Over the course of the present study, rat models of HE were induced and offered a high-protein diet, likely to stimulate ammonia in the colon. These models were simultaneously administered varying levels of MBG-RCA via the intragastric route through oral administration in order to observe the effect on ammonia levels in the blood and intestine. A decrease in these levels could indicate the potential for a future clinically relevant treatment capable of preventing and treating $\mathrm{HE}$ in rats and, in the future, even human subjects. The development of a novel, clinically applicable agent for the oral elimination of intestinal ammonia may revolutionize the treatment of HE.

\section{Materials and methods}

Animal care. The experimental protocols were approved by the Animal Care and Use Committee of Dalian Medical University (Dalian, Liaoning, China) in accordance with guidelines established by the Canadian Council on Animal Care.

A total of 80 male Sprague Dawley rats (250-300 g, 10 weeks of age) were purchased from the Animal Care and Use Committee of Dalian Medical University. Rats were well developed and exhibited no health-related abnormalities or previous pathological conditions. Rats were subsequently divided into 20 A-type stainless steel cages with a sink and a manger. A maximum of five rats were housed in each cage and marked accordingly. Rats were fed common granular fodder containing protein $(23 \%)$, fat $(4.7 \%)$, sodium $(0.24 \%)$ and tap water. The temperature was maintained at $17-24^{\circ} \mathrm{C}$, and rats were subjected to a day-night light cycle reflecting a normal circadian rhythm.

All animal testing was conducted in the laboratory of the College of Pharmacy of Dalian Medical University. The experimental protocols were approved by the Animal Care and Use Committee of Dalian Medical University in accordance with guidelines established by the Canadian Council on Animal Care. 
Reagents. MBG-RCA was prepared using a formulation containing Tween-80 (surfactant), ethylene glycol (cosurfactant), dimethyl silicone oil (external oil phase), an ammonia absorbent with a solution of $30 \%$ acetic acid and $0.9 \%$ sodium chloride (internal phase) and sodium alginate by the Membrane Science and Technology Center at the Dalian University of Technology (21). Lactulose was produced by the Dandong Rehabilitation Pharmaceutical Co. Ltd. (production lot H10890057; Dandong, Liaoning, China) at a concentration of 5\%. Thioacetamide (TAA) was produced by Shanghai Chemical Reagent Company (production lot 920419; Shanghai, China). ALT and TBIL reagent kits were purchased from Nanjing Jiancheng Bioengineering Institute (Nanjing, Jiangsu, China). All materials used were of the highest reagent grade, AR.

Animal model grouping. The eighty rats were divided at random into eight groups of ten. The result was eight distinct groups, consisting of four control groups (normal, blank, lactulose and acetic acid), an HE group, and three MBG-RCA therapeutic groups by dosage (high, medium and low doses of 15,10 and $5 \mathrm{ml} / \mathrm{kg}$ MBG-RCA, respectively). The normal control and blank control groups were fed a normal diet. Conversely, the HE group, acetic acid and lactulose control groups, and the therapeutic MBG-RCA groups were fed a high-protein diet (with $20 \%$ casein added to the normal diet). The rats in the HE group, acetic acid and lactulose control groups, and the MBG-RCA therapeutic groups were prepared as HE models according to the method proposed by Li et al (32). In this method, HE rat models are induced by intraperitoneal injection of TAA $(350 \mathrm{mg} / \mathrm{kg} \cdot \mathrm{day})$ for three consecutive days.

Treatment administrations were conducted once a day over the course of four days. Two hours prior to model establishment, therapeutic MBG-RCA groups were administered $15 \mathrm{ml} / \mathrm{kg}$ (high), $10 \mathrm{ml} / \mathrm{kg}$ (medium) and $5 \mathrm{ml} / \mathrm{kg}$ (low) doses of MBG-RCA. The blank control group was administered equivalent blank microemulsion-based gels. The lactulose control group was administered an equal volume of lactulose $(10 \mathrm{ml} / \mathrm{kg})$. The normal control and HE groups were administered equal volumes of normal (0.9\%) saline. Finally, the acetic acid control group was administered acetic acid solution $(1.15 \%)$. All administrations were conducted using similar tools under similar conditions at a single laboratory setting.

Sample collection. After four days (four doses) rats were consecutively weighed and subsequently placed in an ether anesthesia induction box for $\sim 3 \mathrm{~min}$ in order to induce anesthesia. Rats were removed after full anesthesia had been induced, and $1 \mathrm{ml}$ of blood was drawn from the angular vein of each subject. Simultaneously, abdominal skin was disinfected and the abdominal cavity was opened to expose the inferior vena cava (IVC). Blood $(1.5 \mathrm{ml})$ was drawn from the IVC using a 5-ml syringe. The syringe needle was sealed after drawing blood to prevent ammonia leakage. The sample was immediately placed in a vacuum vessel containing ethylenediamine tetra-acetic acid and centrifuged at $999 \mathrm{x} \mathrm{g}$ for $5 \mathrm{~min}$ at $10-15^{\circ} \mathrm{C}$. The mesentery was separated and the cecum was resected following ligation of both ends, and the excised tissue was placed in a vessel filled with normal $(0.9 \%)$ saline. The colonic wall was cut to release the contents and capped synchronously. The sample was stored at $4^{\circ} \mathrm{C}$ overnight. Supernatant fluid was obtained following centrifugation at a time, temperature and speed consistent with those used for the blood sample.

Subsequently, rats were sacrificed and their livers were truncated for embedding in formalin (10\%) and fixation with neutral balsam.

Liver function analyses. Blood samples were used to evaluate liver function using ALT and TBIL reagent kits (Nanjing Jiancheng Bioengineering Institute). The principle of detection was the enzyme-linked immunosorbent assay and the diazo method, and testing was conducted according to the instructions provided by the manufacturer.

Pathological sections of livers were observed with hematoxylin and eosin (H\&E) staining. Resultant observations included the structure of hepatic cords and sinuses; shapes of liver cells; number, appearance and location of the cell nuclei; and infiltration of inflammatory cells, edema, necrosis or other abnormalities, if present.

Blood ammonia level analyses. Blood samples taken from the IVC were analyzed for ammonia content. Blood ammonia was detected using an Automatic Dry-type Biochemical Analyzer (Johnson \& Johnson, New Brunswick, NJ, USA). The entire process was completed within $30 \mathrm{~min}$ of collection from the specimens and blood was stored in sealed vials to prevent ammonia leakage prior to testing.

Additionally, intestinal ammonia in the supernatant fluid collected from the excised cecum tissue was detected using the Automatic Dry-type Biochemical Analyzer. The data were corrected according to the weight of the excised tissue.

Measurement of ethological indicators. The general condition of rats, including mental state, sleeping time (lethargic time) and mortality was observed using the Infrared Night Vision Surveillance System (Jinan dimensional Century Technology Co., Ltd., Jinan, Shandong, China), consisting of community monitoring by video capture cards embedded in cage-mounted digital devices (Guangzhou Ao Qiman Electronic Technology Co., Ltd., Guangzhou, Guangdong, China). Food intake and sleep duration were determined using electronic scales and observational methods, respectively, and recorded once daily. Initial and final rat body weights were noted on the first and fourth day using electronic scales. The grading score of $\mathrm{HE}$ was analyzed according to the standards by Zimmerman et al (33). In brief, the gradings were as follows: Level 0, normal; Level I, drowsiness, slow responses, reduced locomotor activity and normal reflexes; Level II, ataxia but with normal reflexes; Level III, gradual disappearance of reflexes; and Level IV, lack of corneal reflex/unconsciousness.

Electroencephalographic analyses. On the fourth day, prior to sample collection, rat subjects were anesthetized using pelltobarbitalum natricum $(50 \mathrm{mg} / \mathrm{kg})$ and fixed on a stereotaxic instrument $\sim 30 \mathrm{~min}$ afterwards. The rats were awake for all subsequent tests. EEG was applied to all rats using the 
Biological Function of Experimental System (model BL-420F; Chengdu Taimeng Technology Co., Ltd., Chengdu, China). EEG was traced for 10 min with a time constant of 0.3 and a high-frequency filter setting of $100 \mathrm{~Hz}$. Frequency and amplitude were indicated in the EEG, and $\alpha$ waves were primarily on top of the brains of normal rats (frequency, 8-13 times/sec; voltage, 50-100 $\mu \mathrm{V}$ ). Mild EEG abnormalities were displayed as low-rhythm $\alpha$ waves mingled with $\theta$ waves (frequency primarily at 8-11 times/sec, voltage at 150-250 $\mu \mathrm{V}$ ). Severe EEG abnormalities were revealed as extensive wide and deep $\delta$ waves (frequency, 5-7 times/sec; voltage 160-280 $\mu \mathrm{V}$ ). All rats were awake for $\sim 4-6 \mathrm{~h}$.

Statistical analyses. Data analyses were conducted using SPSS 11.5 software (Chicago, IL, USA). Measurement data are presented as the mean \pm standard deviation, matching normal distribution. One-factor analysis of variance was used for group comparison whereas least square difference testing was applied for pairwise comparisons. The rank-sum test was used to assess ranked data. Rank correlation was used for correlation analyses. The $\chi^{2}$ test was used for comparison of enumeration data. $\mathrm{P}<0.05$ was considered to indicate a statistically significant difference.

\section{Results}

Qualitative condition of subjects. Compared with the normal control group, no abnormality was observed in the blank control group. Rats in the HE group, however, exhibited visual signs of depressed activity levels, a hunched or irregular stature, and jittery or inconsistent movement patterns. Additionally, these subjects exhibited a visually apparent decreased desire for foraging, and two subjects developed hemorrhagia nasalis. Therapeutic groups, as well as the acetic acid and lactulose control groups, exhibited reduced or milder manifestations of the above symptoms, generally concurrent with the increasing dosage of MBG-RCA in the therapeutic groups.

Control group results. No significant differences between the normal control group and blank control group were observed in terms of food intake, sleep duration, weight change, HE grading score, ALT and TBIL levels, blood ammonia levels or intestinal ammonia levels $(\mathrm{P}>0.05)$. Furthermore, there were no mortalities in the normal or blank control groups $(100 \%$ survival). The EEG of the normal and blank control groups showed no abnormality and presented mainly as $\alpha$ waves (frequency, 8-13 times/sec; voltage, 50-100 $\mu \mathrm{V}$ ). H\&E staining in the excised hepatic tissue of the normal and blank control groups revealed no obvious abnormalities.

Food intake. The food intake amount in each therapeutic group and the lactulose and acetic acid control groups exhibited recovery compared with the HE group. The HE group food intake was observed to be $51 \pm 18 \mathrm{~g} / \mathrm{kg}$-day. The high-, mediumand low-dose MBG-RCA therapeutic groups exhibited a greatly increased observed food intake of $92 \pm 15(\mathrm{P}=0.000)$, $92 \pm 16(\mathrm{P}=0.000)$ and $77 \pm 20 \mathrm{~g} / \mathrm{kg} \cdot$ day $(\mathrm{P}=0.018)$, respectively. The lactulose and acetic acid control groups demonstrated a moderate food intake of $75 \pm 23(\mathrm{P}=0.024)$ and $72 \pm 20 \mathrm{~g} / \mathrm{kg} \cdot$ day $(\mathrm{P}=0.022)$, respectively. Food intake amounts in the high-dose
MBG-RCA group were significantly higher than those in the lactulose and acetic acid control groups by 122 and 129\%, respectively. Compared with the medium-dose MBG-RCA group, food intake in the acetic acid and lactulose control groups was significantly lower, by 78 and $82 \%$, respectively. No significant differences, however, were noted among the acetic acid and lactulose control groups and low-dose MBG-RCA groups (Table I). Statistically, no clear correlation was observed between the food intake and dosage of MBG-RCA; however, the level of food intake was similarly elevated in the mediumand high-dosage therapeutic groups (Fig. 1).

Sleep duration. In comparison with the HE sleep duration of $20 \pm 2 \mathrm{~h} / 24 \mathrm{~h}$, the therapeutic groups were observed to have decreased sleep durations of $12 \pm 4.0(\mathrm{P}=0.001), 13 \pm 3$ $(\mathrm{P}=0.001)$ and $16 \pm 4 \mathrm{~h} / 24 \mathrm{~h}(\mathrm{P}=0.006)$ in the high, medium and low MBG-RCA dosage groups, respectively. The lactulose and acetic acid control groups exhibited sleep durations of $16 \pm 5(\mathrm{P}=0.030)$ and $17 \pm 5 \mathrm{~h} / 24 \mathrm{~h}(\mathrm{P}=0.037)$, respectively. The sleep duration in the high-dose MBG-RCA group was significantly shorter than that observed in the lactulose and acetic acid control groups, by 74 and $73 \%$, respectively. Conversely, when compared with the medium-dose MBG-RCA group, the lactulose and acetic acid control group demonstrated increased sleep durations, by 130 and $132 \%$, respectively. No significant differences, however, were noted among the acetic acid and lactulose control groups and the low-dose MBG-RCA group. The observed sleep duration in high- and medium-dose MBG-RCA groups was shorter than that observed in the low-dose MBG-RCA group by 75 and $78 \%$, respectively. These results indicate a negative correlation between sleep duration and increasing dosages of MBG-RCA (Table I and Fig. 1).

Weight changes. Rats in the HE group exhibited a weight change of $37 \pm 8 \mathrm{~g}$. The high-, medium- and low-dosage MBG-RCA groups exhibited weight changes of $14 \pm 4 \quad(\mathrm{P}=0.000)$, $15 \pm 5(\mathrm{P}=0.000)$ and $37 \pm 8 \mathrm{~g}(\mathrm{P}=0.001)$, respectively. The lactulose and acetic acid control groups exhibited weight changes of $22 \pm 4(\mathrm{P}=0.001)$ and $21 \pm 2 \mathrm{~g}(\mathrm{P}=0.001)$, respectively. The weight decrease in the high-dose MBG-RCA group was significantly lower than that observed in the lactulose and acetic acid control groups, by 65 and $67 \%$, respectively. Compared with the medium-dose MBG-RCA group, the lactulose and acetic acid control groups demonstrated decreased weight changes of 70 and $71 \%$, respectively. No significant differences among the acetic acid and lactulose control groups and the low-dose MBG-RCA group were observed. Weight decreases in the high- and medium-dose MBG-RCA groups were significantly lower than that observed in the low-dose MBG-RCA group, by 38 and $41 \%$, respectively. Accordingly, the results indicate a negative correlation between the decreasing change in weight and the increasing dosage of MBG-RCA (Table I and Fig. 1).

HE grading score. Rats in each therapeutic group demonstrated higher $\mathrm{HE}$ grading scores compared with the $\mathrm{HE}$ group score of $2.7 \pm 0.9$, demonstrating high, medium and low $\mathrm{HE}$ grading scores of $1.1 \pm 0.3(\mathrm{P}=0.001), 1.2 \pm 0.4(\mathrm{P}=0.004)$ and $1.8 \pm 0.8(\mathrm{P}=0.188)$, respectively. The lactulose and acetic acid control groups exhibited HE grading scores of $1.9 \pm 0.7$ $(\mathrm{P}=0.000)$ and $2.0 \pm 0.8(\mathrm{P}=0.225)$, respectively. The $\mathrm{HE}$ 
Table I. Comparisons of associated indicators among rat groups (mean \pm standard deviation).

\begin{tabular}{|c|c|c|c|c|c|c|}
\hline Indicators & $\mathrm{C}$ & $\mathrm{D}$ & $\mathrm{E}$ & $\mathrm{F}$ & G & $\mathrm{H}$ \\
\hline Food intake (g/kg·day) & $54.0 \pm 18.0^{\mathrm{a}}$ & $77.0 \pm 20.0$ & $92.0 \pm 16.0^{\mathrm{d}}$ & $92.0 \pm 15.0^{\mathrm{d}}$ & $72.0 \pm 20.0$ & $75.0 \pm 23.0$ \\
\hline Sleep duration $(\mathrm{h} / 24 \mathrm{~h})$ & $20.0 \pm 2.0^{\mathrm{a}, \mathrm{d}}$ & $16.0 \pm 4.0$ & $13.0 \pm 3.0^{\mathrm{d}}$ & $12.0 \pm 4.0^{\mathrm{a}, \mathrm{d}}$ & $17.0 \pm 5.0$ & $16.0 \pm 5.0$ \\
\hline Weight changes (g) & $37.0 \pm 8.0^{\mathrm{c}, \mathrm{d}}$ & $37.0 \pm 8.0^{\mathrm{c}, \mathrm{d}}$ & $15.0 \pm 5.0^{\mathrm{b}, \mathrm{d}}$ & $14.0 \pm 4.0^{\mathrm{c}, \mathrm{d}}$ & $21.0 \pm 2.0$ & $22.0 \pm 4.0$ \\
\hline HE grading score & $2.7 \pm 0.9^{\mathrm{a}}$ & $1.8 \pm 0.8$ & $1.2 \pm 0.4^{\mathrm{a}}$ & $1.1 \pm 0.3^{\mathrm{b}, \mathrm{d}}$ & $2.0 \pm 0.8$ & $1.9 \pm 0.7$ \\
\hline Blood ammonia $(\mu \mathrm{mol} / 1)$ & $286.0 \pm 27.0^{\mathrm{c}, \mathrm{d}}$ & $243.0 \pm 37.0$ & $209.0 \pm 28.0^{\mathrm{c}, \mathrm{d}}$ & $199.0 \pm 42.0^{\mathrm{a}, \mathrm{d}}$ & $252.0 \pm 28.0$ & $246.0 \pm 33.0$ \\
\hline Intestinal ammonia $(\mu \mathrm{mol} / \mathrm{l})$ & $410.0 \pm 56.0^{\mathrm{a}, \mathrm{d}}$ & $317.0 \pm 47.0^{\mathrm{d}}$ & $270.0 \pm 88.0$ & $251.0 \pm 69.0^{\mathrm{a}}$ & $246.0 \pm 70.0$ & $336.0 \pm 72.0$ \\
\hline $\operatorname{ALT}(\mathrm{U} / \mathrm{l})$ & $252.0 \pm 22.0^{\mathrm{d}}$ & $219.0 \pm 23.0$ & $202.0 \pm 22.0^{\mathrm{a}}$ & $196.0 \pm 24.0^{\mathrm{a}, \mathrm{d}}$ & $226.0 \pm 18.0$ & $223.0 \pm 20.0$ \\
\hline TBIL $(\mu \mathrm{mol} / \mathrm{l})$ & $55.0 \pm 12.0^{\mathrm{a}}$ & $45.0 \pm 10.0$ & $38.0 \pm 5.0^{\mathrm{b}, \mathrm{d}}$ & $36.0 \pm 6.0^{\mathrm{b}, \mathrm{d}}$ & $48.0 \pm 10.0$ & $46.0 \pm 5.0$ \\
\hline
\end{tabular}

${ }^{\mathrm{a}} \mathrm{P}<0.05,{ }^{\mathrm{b}} \mathrm{P}<0.01$ and ${ }^{\mathrm{c}} \mathrm{P}<0.001$, compared with the lactulose control group; ${ }^{\mathrm{d}} \mathrm{P}<0.05$ compared with the acetic acid control group; $\mathrm{C}, \mathrm{HE}$ group; D, low-dose MBG-RCA group; E, medium-dose MBG-RCA group; F, high-dose MBG-RCA group; G, acetic acid control group; H, lactulose control group; HE, hepatic encephalopathy; ALT, alanine aminotransferase; TBIL, total bilirubin; MBG-RCA, microemulsion-based gel for the removal of colonic ammonia.
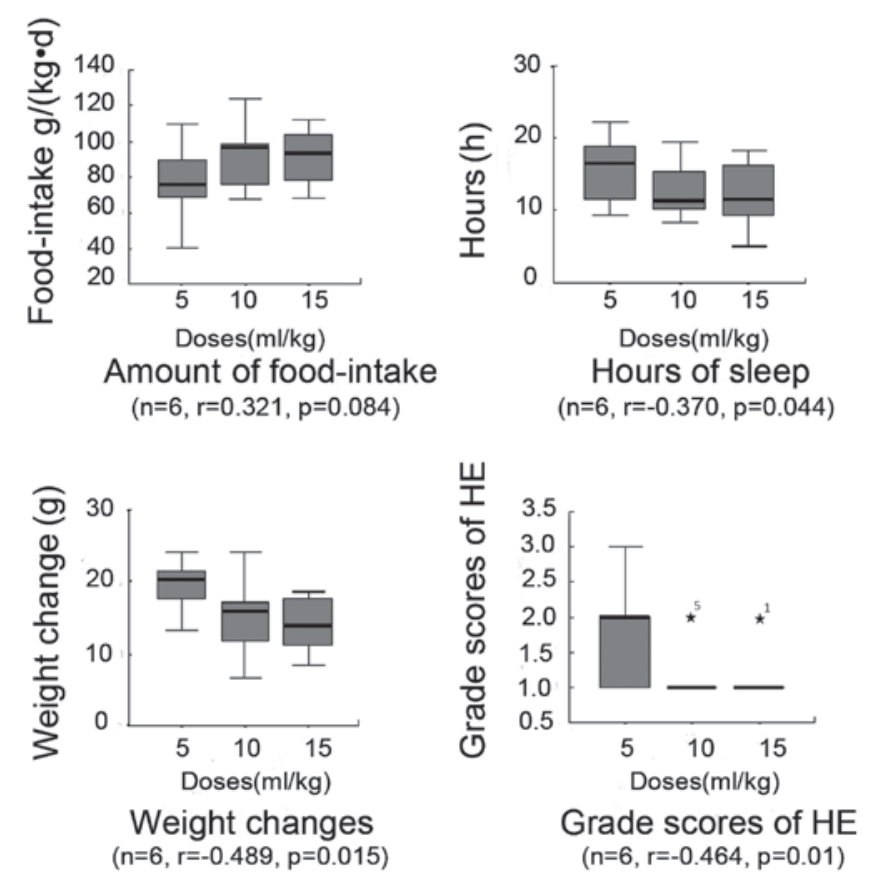

Figure 1. Correlation between dosage of microemulsion-based gel for the removal of colonic ammonia and food intake, sleep duration, weight change and hepatic encephalopathy (HE) scoring grade in rat groups $(n=6) .{ }^{* 5}$ and $* 1$ represent outliers.

grading score in the high-dose MBG-RCA group was significantly higher than those observed in the lactulose and acetic acid control groups by 58 and 55\%, respectively. Compared with the medium-dose MBG-RCA group, the lactulose and acetic acid control groups demonstrated increased HE scores by 70 and $74 \%$, respectively. No significant differences among the acetic acid and lactulose control groups and the low-dose MBG-RCA group were noted. Furthermore, the HE grading scores in the high- and medium-dose MBG-RCA groups, as well as in the medium- and low-dose MBG-RCA groups, revealed no significant differences. HE grading scores of the high-dose MBG-RCA group were lower than those of the low-dose MBG-RCA group by $61 \%$. Thus, the results suggest

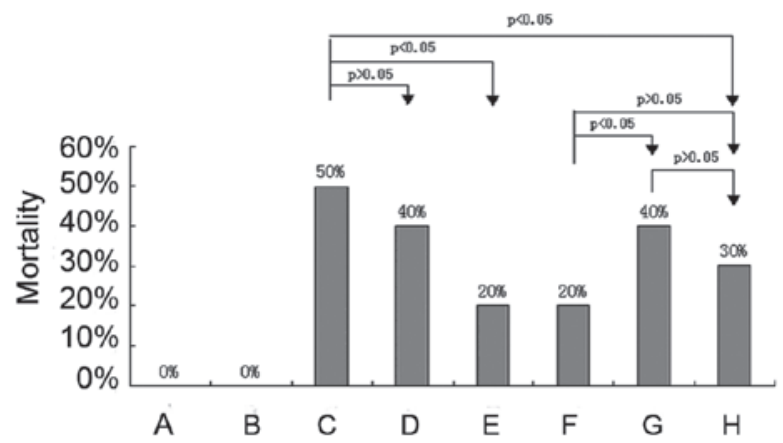

Figure 2. Comparison of mortality among rat groups. (A) Normal control group; (B) blank control group; (C) hepatic encephalopathy group; (D) low-dose microemulsion-based gel for the removal of colonic ammonia (MBG-RCA) group; (E) medium-dose MBG-RCA group; (F) high-dose MBG-RCA group; (G) acetic acid control group; (H) lactulose control group.

a negative correlation between weight decrease and dosage of MBG-RCA (Table I and Fig. 1).

Mortality. The prevalence of mortality in the HE group and in the rats receiving therapeutic high, medium and low doses of MBG-RCA was 50, 20, 20 and 40\%, respectively. The lactulose and acetic acid control group treatments exhibited mortality rates of 30 and $40 \%$, respectively. Rats in the highand medium-dose MBG-RCA groups as well as those in the lactulose control group revealed a statistically significant lower mortality than those in the HE group, although the low-dose MBG-RCA and acetic acid control groups exhibited no significant difference from the HE group. The mortality of each group is shown in Fig. 2.

EEG. EEGs of the HE group were notably abnormal, revealing extensive wide and deep $\delta$ waves (frequency, 5-7 times/sec; voltage, 160-280 $\mu \mathrm{V}$ ). Medium- and high-dose MBG-RCA groups revealed low-to-moderate EEG abnormalities characterized by a mixture of low-rhythm $\alpha$ waves and $\theta$ waves (frequency mainly at 8-11 times/sec; voltage, 150-250 $\mu \mathrm{V}$ ). In the acetic acid and lactulose control groups, as well as the 
A

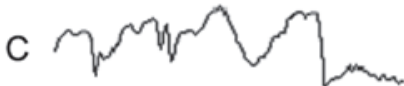

E
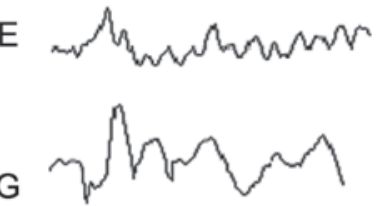

$50 \mu \mathrm{V} \frac{\mathrm{L}}{12 \mathrm{~mm} / \mathrm{s}}$

Figure 3. Changes in electroencephalography among rat groups. (A) Normal control group; (B) blank control group; (C) hepatic encephalopathy group; (D) low-dose microemulsion-based gel for the removal of colonic ammonia (MBG-RCA) group; (E) medium-dose MBG-RCA group; (F) high-dose MBG-RCA group; (G) acetic acid control group; (H) lactulose control group

low-dose MBG-RCA group, EEGs were observed to be moderately abnormal, presenting abnormal $\theta$ waves (frequency, 7-9 times/sec; voltage, 130-200 $\mu \mathrm{V}$ ) as shown in Fig. 3.

Blood ammonia level. Rats receiving therapeutic treatments exhibited reduced blood ammonia levels compared with the HE group, which had a blood ammonia level of $286 \pm 27 \mu \mathrm{mol} / 1$. The high-, medium- and low-dose MBG-RCA groups exhibited blood ammonia levels of $199 \pm 42(\mathrm{P}=0.000)$, $209 \pm 28(\mathrm{P}=0.001)$ and $243 \pm 37 \mu \mathrm{mol} / 1(\mathrm{P}=0.054)$, respectively. The lactulose and acetic acid control groups exhibited blood ammonia levels of $246 \pm 33(\mathrm{P}=0.027)$ and $252 \pm 28 \mu \mathrm{mol} / \mathrm{l}$ $(\mathrm{P}=0.155)$, respectively. Blood ammonia in the high-dose MBG-RCA group was significantly lower than that observed in the lactulose and acetic acid control groups, by 81 and $80 \%$, respectively. Compared with the medium-dose MBG-RCA group, the lactulose and acetic acid control groups exhibited increasing levels of blood ammonia by 118 and $121 \%$, respectively. No significant differences among the acetic acid and lactulose control groups and the low-dose MBG-RCA group were observed. Table I shows the blood ammonia levels in each group. The blood ammonia levels observed in the high- and medium-dose MBG-RCA groups were significantly lower than that observed in the low-dose MBG-RCA group, indicating a negative correlation between the blood ammonia and the increasing dose of MBG-RCA (Fig. 4).

Intestinal ammonia level. Rats in the therapeutic treatment groups were observed to have lower levels of intestinal ammonia compared with the HE group, with a level of $410 \pm 56 \mu \mathrm{mol} / 1$. The high-, medium- and low-dose MBG-RCA groups exhibited levels of $251 \pm 69(\mathrm{P}=0.001), 270 \pm 88(\mathrm{P}=0.003)$ and $317 \pm 47 \mu \mathrm{mol} / 1(\mathrm{P}=0.013)$, respectively. The lactulose and acetic acid control groups exhibited levels of $336 \pm 72$ $(\mathrm{P}=0.11)$ and $246 \pm 70 \mu \mathrm{mol} / 1(\mathrm{P}=0.051)$, respectively. Intestinal ammonia in the high-dose MBG-RCA group was observed to be significantly lower than in the lactulose and acetic acid control groups, by 75 and $102 \%$, respectively. Compared with the medium-dose MBG-RCA group, the lactulose and acetic acid control groups demonstrated higher amounts by 124 and $91 \%$, respectively. No significant differences among the acetic
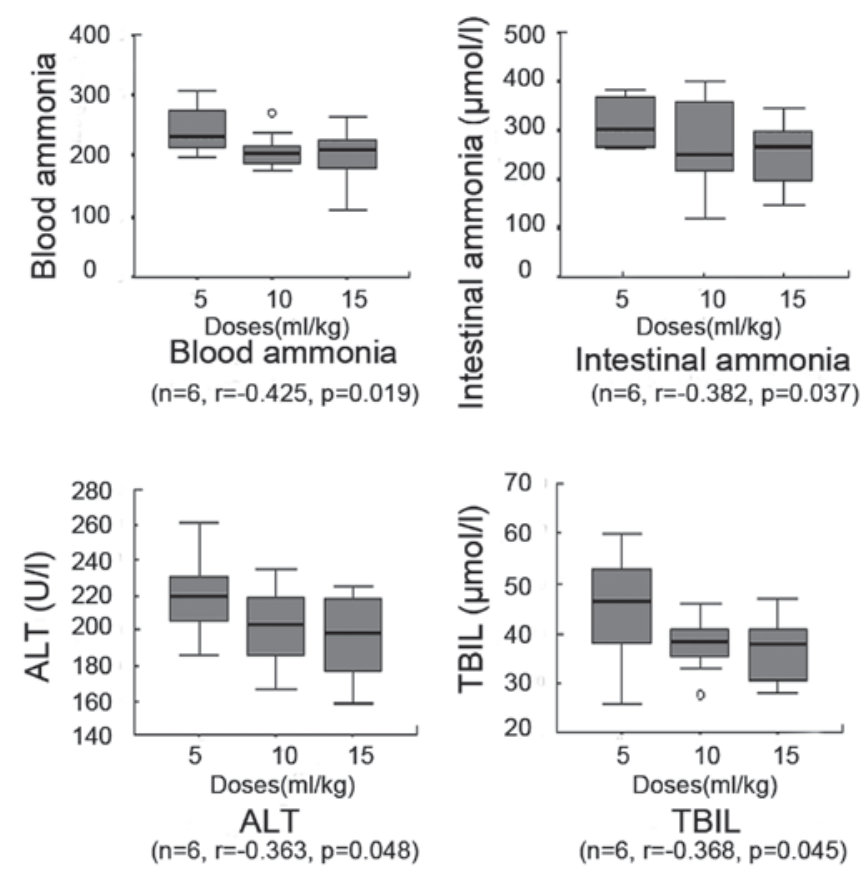

Figure 4. Correlation between dosage of microemulsion-based gel for the removal of colonic ammonia and the level of blood ammonia, intestinal ammonia, alanine aminotransferase (ALT) and total bilirubin (TBIL) in rat groups $(n=6)$. Circles indicate outliers.

acid and lactulose control groups and the low-dose MBG-RCA group were observed. Table I shows details of the intestinal ammonia levels in each group. The correlation between blood ammonia and the administered dose of MBG-RCA was negative (Fig. 4).

ALT and TBIL levels. Rats in the therapeutic treatment groups exhibited lower ALT levels compared with the HE group, with an ALT level of $252 \pm 22 \mathrm{U} / 1$ and a TBIL level of $55 \pm 12 \mu \mathrm{mol} / 1$. The high-, medium- and low-dose MBG-RCA groups exhibited ALT levels of $196 \pm 24(\mathrm{P}=0.001), 202 \pm 22(\mathrm{P}=0.002)$ and $219 \pm 23 \mathrm{U} / 1(\mathrm{P}=0.01)$, respectively, and TBIL levels of $36 \pm 6(\mathrm{P}=0.002), 38 \pm 5(\mathrm{P}=0.002)$ and $45 \pm 10 \mu \mathrm{mol} / \mathrm{l}(\mathrm{P}=0.138)$, respectively. Lactulose and acetic acid control groups exhibited ALT levels of $222 \pm 20(\mathrm{P}=0.038)$ and $226 \pm 18 \mathrm{U} / 1$ $(\mathrm{P}=0.045)$, respectively, and TBIL levels of $46 \pm 5(\mathrm{P}=0.041)$ and $48 \pm 10 \mu \mathrm{mol} / 1(\mathrm{P}=0.325)$, respectively. The ALT and TBIL levels in the high-dose MBG-RCA group were significantly lower than those in the lactulose and acetic acid control groups, by 88 and $87 \%$ for ALT and by 80 and $77 \%$ for TBIL, respectively. Compared with the medium-dose MBG-RCA group ALT and TBIL levels, the lactulose and acetic acid control groups demonstrated elevated levels, by 110 and $112 \%$ for ALT and by 121 and $126 \%$ for TBIL, respectively. No significant differences were observed among the acetic acid and lactulose control groups and the low-dose MBG-RCA groups in ALT or TBIL levels. Finally, ALT and TBIL levels in the high- and medium-dose MBG-RCA groups as well as in the mediumand low-dose MBG-RCA groups demonstrated no significant differences. Conversely, the high-dose MBG-RCA group demonstrated lower levels than the low-dose MBG-RCA group for both ALT and TBIL, as shown in Fig. 4. The correlation between the ALT and TBIL levels and the increasing dosage 

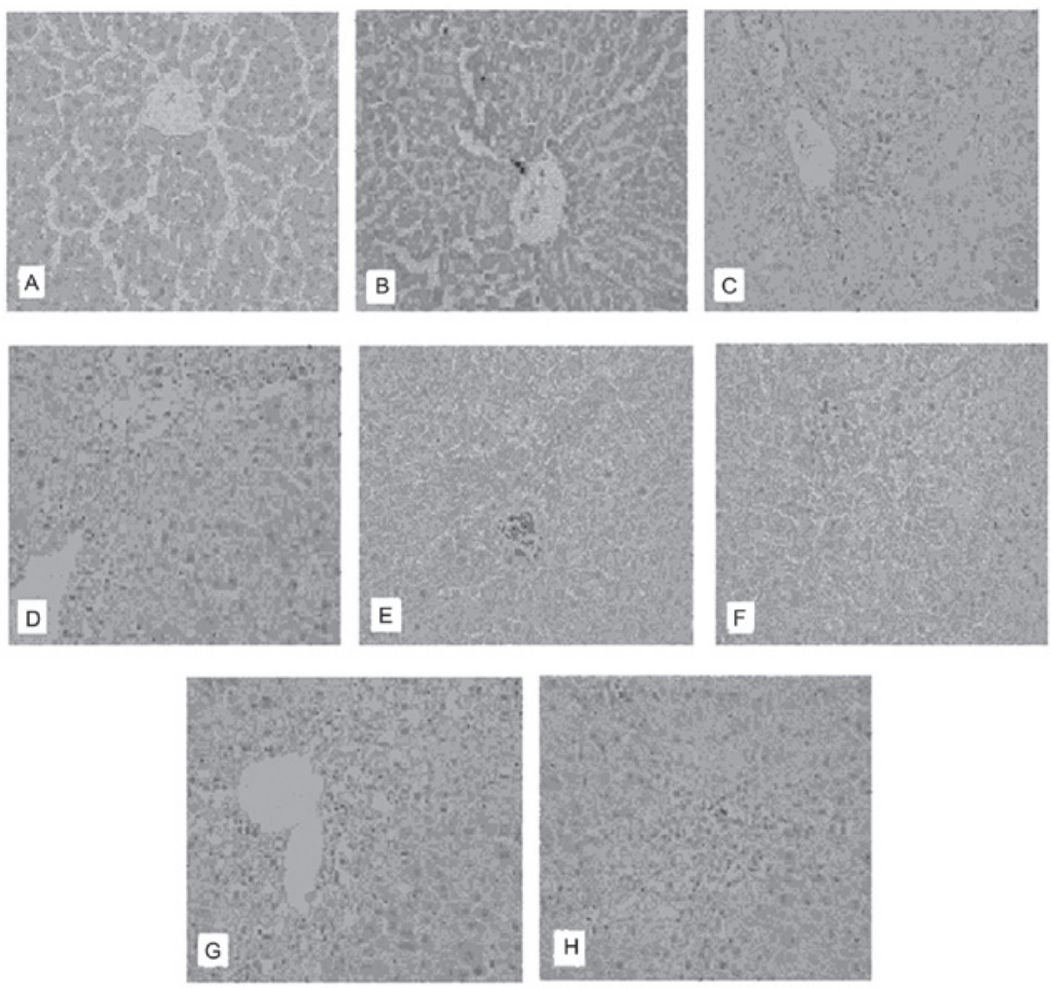

Figure 5. Pathological changes in liver tissues in rat groups (hematoxylin and eosin; magnification, x200). (A) Normal control group; (B) blank control group; (C) hepatic encephalopathy group; (D) low-dose microemulsion-based gel for the removal of colonic ammonia (MBG-RCA) group; (E) medium-dose MBG-RCA group; (F) high-dose MBG-RCA group; (G) acetic acid control group; (H) lactulose control group.

of MBG-RCA was determined to be negative based on these findings (Table I and Fig. 4).

Liver pathology. The structure of the excised hepatic tissue examined with $H \& E$ staining exhibited unclear results in the HE group, with a characteristic disordered architecture of the hepatic lobule containing edema and ballooning degeneration. Necrosis coupled with the presence of infiltrating inflammation cells was extensively observed. The portal area was notably infiltrated by inflammatory cells, and the liver sinusoids were extended and congested. The acetic acid and lactulose control groups, as well as the therapeutic groups, exhibited varying levels of the pathological changes described above (Fig. 5).

\section{Discussion}

Rat models provide a viable point of reference for the in vivo treatment of HE by administration of oral medications, including the $\mathrm{pH}$-sensitive microemulsion gel proposed in this study. Rat models of type A HE, as modeled in the current study, were induced by intraperitoneal injection of TAA, a method known for its outstanding repeatability, shorter completion time and higher success rate compared with other established methods of HE treatment. Furthermore, the model has been widely accepted internationally due to its similarity with human liver damage $(34,35)$. Thus, this study provides an initial step in the development of a novel and clinically viable treatment and prevention of HE in humans.

MBG-RCA molecules are designed to survive the $\mathrm{pH}$ variation of normal oral administration without releasing their ammonia-reducing contents until reaching the specific $\mathrm{pH}$ levels found in the colon. In order to exclude the possibility of the influence of the microemulsion molecules on rats directly, the normal control group was compared with the blank control group. The treatment effect of MBG-RCA on removing colonic ammonia was further verified by establishing therapeutic controls, including acetic acid (enteroclysis for acidifying the colonic tract) and lactulose (commonly used to reduce colonic ammonia). Furthermore, high, medium and low doses of MBG-RCA were applied to observe the dose correlation.

In the present study, MBG-RCA was shown to reduce blood ammonia by removing intestinal ammonia directly from the colon, its primary center of production. The results of ammonia removal included significant improvements in sleep duration (including stupor), neural reflex, EEG, food intake, weight and mortality in HE model rats. High- and medium-level dosages of MBG-RCA demonstrated superior results to treatment with either acetic acid, lactulose or low dosages of MBG-RCA, indicating a positive correlation of symptom cessation with increasing dosage of MBG-RCA. Notably, high and medium MBG-RCA dosages were shown to be more effective than the lactulose treatments commonly used in clinical settings. These treatments also have the benefit of being able to directly remove intestinal ammonia without the involvement of colonic bacteria or enzymes, producing fewer restrictions compared with contemporary lactulose treatments.

Acetic acid may also be influenced by the $\mathrm{pH}$ of the GI tract, and may thus be neutralized, diluted or consumed prior to reaching the colon. This effect is likely responsible for the few documented accounts of oral administration of acetic acid for therapeutic purposes (36). The current study, however, 
indicates that direct oral intake of acetic acid may serve to reduce blood and intestinal levels of ammonia when dosages are sufficient to overcome the ability of the GI tract to neutralize the compound, thus reducing acute liver failure and HE symptoms. The remaining acetic acid that reaches the colon may be enough to acidify the colonic tract, removing ammonia from the body as ammonium ions. However, mortality in the acetic acid control group was relatively high in the present study, with abnormalities including edema common in hepatic cells. These findings are concurrent with studies suggesting the use of acetic acid solution as a coloclyster to treat HE by acidifying the colonic tract (37). This method, however, is not suitable for preventing $\mathrm{HE}$ as it is relatively complex and requires significant patient cooperation during an uncomfortable treatment.

In rat models, food intake and sleep were influenced by MBG-RCA administration, indicating that this treatment is likely to improve the quality of life by increasing food intake and reducing sleep duration. Subjects administered MBG-RCA demonstrated similar mortality rates to those treated with lactulose, and both rates were significantly lower than those observed with low-dose MBG-RCA and acetic acid treatments as well as those observed among the untreated (HE group) subjects. While these results are encouraging, a larger sample size is required to further study the effectiveness and optimal dosage of MBG-RCA treatment. MBG-RCA treatment was shown to produce notably reduced levels of ALT and TBIL, superior to lactulose treatment.

Whether MBG-RCA ameliorates pathological findings remains unknown. As lactulose combined with antibiotic administration has often been suggested to improve liver performance, it is possible that combined treatment may also demonstrate positive effects with microemulsions (38-40). Other drugs that improve the metabolism and increase survival rates by reducing toxin absorption, thus protecting the brain and liver tissues, may also be beneficial when administered together with MBG-RCA $(41,42)$. The optimal mechanism and administration technique for MBG-RCA to enhance liver function requires further study; however, it is likely that the removal of intestinal ammonia is only one aspect of MBG-RCA's potential benefits in HE treatment.

While several indirect methods exist, the majority with significant side effects, no method of directly decreasing the intestinal level of ammonia by oral medication has been developed. The MBG-RCA designed in this experiment is the first to remove intestinal ammonia without the involvement of colonic bacteria or enzymes, reducing side effects and discomfort during treatment. It has the advantages of higher clearance, stability of the GI tract and elimination of ammonia from the body without producing harmful metabolic byproducts.

The MBG-RCA designed in the current study has demonstrated excellent potential for in vivo treatment of HE by direct ammonia reduction; however, numerous questions remain unanswered with regard to the mechanism, action and molecular structure. These issues include optimal surfactant/co-surfactant determination and proportioning, prevention of adverse reactions, and methods to reduce acid wastage during GI transport upon oral administration. Although no enzymes along the course of the GI tract have been determined to influence this molecule, it is yet to be ascertained whether the molecule has an effect on digestive enzyme molecules as it travels through the body. These observations may play a significant role in assessing drug interactions, improving compound stability and reducing side effects. Additional pharmacological research is required to determine the optimal formula for maximal removal of intestinal ammonia with minimal side effects. Although MBG-RCA remains experimental, its novel characteristics merit future investigation as a potential clinical alternative for removal of ammonia in humans.

\section{Acknowledgements}

The current study was supported by the National Natural Science Foundation of China (grant no. 30970886) and the Science and Technology Project of Dalian (grant no. 2008E13SF193).

\section{References}

1. Butterworth RF, Norenberg MD, Felipo V, Ferenci P, Albrecht J and Blei AT; Members of the ISHEN Commission on Experimental Models of HE: Experimental models of hepatic encephalopathy: ISHEN guidelines. Liver Int 29: 783-788, 2009.

2. Huang HC, Chang CC, Wang SS, Chan CY, Lee FY, et al: Pravastatin for thioacetamide-induced hepatic failure and encephalopathy. Eur J Clin Invest 42: 139-145, 2012.

3. Rama Rao KV, Reddy PV, Tong X and Norenberg MD: Brain edema in acute liver failure: inhibition by L-histidine. Am J Pathol 176: 1400-1408, 2010.

4. Butterworth RF: Altered glial-neuronal crosstalk: cornerstone in the pathogenesis of hepatic encephalopathy. Neurochem Int 57: 383-388, 2010.

5. Strekalova OS, Uchaĭkin VF, Ipatova OM, Torkhovskaia TI, Medvedeva NV, et al: Comatose states: etiopathogenesis, experimental studies, treatment of hepatic coma. Biomed Khim 55: 380-396, 2009 (In Russian).

6. Hassanein T, Blei AT, Perry W, Hilsabeck R, Stange J, et al: Performance of the hepatic encephalopathy scoring algorithm in a clinical trial of patients with cirrhosis and severe hepatic encephalopathy. Am J Gastroenterol 104: 1392-1400, 2009.

7. Kochar DK, Agarwal P, Kochar SK, Jain R, Rawat N, et al: Hepatocyte dysfunction and hepatic encephalopathy in Plasmodium falciparum malaria. QJM 96: 505-512, 2003.

8. Bémeur C, Vaquero J, Desjardins P and Butterworth RF: $\mathrm{N}$-acetylcysteine attenuates cerebral complications of non-acetaminophen-induced acute liver failure in mice: antioxidant and anti-inflammatory mechanisms. Metab Brain Dis 25: 241-249, 2010.

9. Sharma P, Sharma BC and Sarin SK: Critical flicker frequency for diagnosis and assessment of recovery from minimal hepatic encephalopathy in patients with cirrhosis. Hepatobiliary Pancreat Dis Int 9: 27-32, 2010.

10. Zafirova $\mathrm{Z}$ and $\mathrm{O}^{\prime}$ Connor M: Hepatic encephalopathy: current management strategies and treatment, including management and monitoring of cerebral edema and intracranial hypertension in fulminant hepatic failure. Curr Opin Anaesthesiol 23: 121-127, 2010.

11. Al Sibae MR and McGuire BM: Current trends in the treatment of hepatic encephalopathy. Ther Clin Risk Manag 5: 617-626, 2009.

12. Zheng Z, Li X, Li Z and Ma X: Artificial and bioartificial liver support systems for acute and acute-on-chronic hepatic failure: A meta-analysis and meta-regression. Exp Ther Med 6: 929-936, 2013.

13. Choi JW, Yoon KT, Park JY, Kim JK, Ahn SH, et al: Usefulness and safety of extracorporeal liver support therapy using MARSR for patients with liver failure: a preliminary report. Korean J Gastroenterol 54: 28-35, 2009 (In Korean).

14. Phongsamran PV, Kim JW, Cupo Abbott J and Rosenblatt A: Pharmacotherapy for hepatic encephalopathy. Drugs 70 : 1131-1148, 2009.

15. Sharma P and Sharma BC: Disaccharides in the treatment of hepatic encephalopathy. Metab Brain Dis 28: 313-320, 2013.

16. Sharma BC, Sharma P, Agrawal A and Sarin SK: Secondary prophylaxis of hepatic encephalopathy: an open-label randomized controlled trial of lactulose versus placebo. Gastroenterology 137: 885-891, 891.e1, 2009. 
17. Malaguarnera M, Gargante MP, Malaguarnera G, Salmeri M, Mastrojeni S, et al: Bifidobacterium combined with fructo-oligosaccharide versus lactulose in the treatment of patients with hepatic encephalopathy. Eur J Gastroenterol Hepatol 22: 199-206, 2010.

18. Weber FL Jr: Effects of lactulose on nitrogen metabolism. Scand J Gastroenterol Suppl 222: 83-87, 1997.

19. Sotelo N, de los Angeles Durazo M, Gonzalez A and Dhanakotti N: Early treatment with $\mathrm{N}$-acetylcysteine in children with acute liver failure secondary to hepatitis A. Ann Hepatol 8: 353-358, 2009.

20. Sundaram V and Shaikh OS: Hepatic encephalopathy: pathophysiology and emerging therapies. Med Clin North Am 93 819-836, 2009.

21. Wang AH, Duan ZJ, Tian G, Lu D, Zhang WJ, et al: The addition of a $\mathrm{pH}$-sensitive gel improves microemulsion stability for the targeted removal of colonic ammonia. BMC Gastroenterol 11: $50,2011$.

22. Tønnesen HH and Karlsen J: Alginate in drug delivery systems. Drug Dev Ind Pharm 28: 621-630, 2002.

23. Pan MD, Li JC, Lin Q, Wang XH and Wang LH: Progress on sodium alginate application to drug controlled release. China Pharmaceuticals 17: 3-5, 2008 (In Chinese).

24. Chen SC, Wu YC, Mi FL, et al: A novel pH-sensitive hydrogel composed of $\mathrm{N}, \mathrm{O}$-carboxymethyl chitosan and alginate crosslinked by genipin for protein drug delivery. J Control Release 96 285-300, 2004

25. Moroni A, Drefko W and Thone G: Formulations of zero-order, $\mathrm{pH}$-dependent, sustained release matrix systems by ionotropic gelation of alginate-containing mixtures. Drug Dev Ind Pharm 37: 216-224, 2011.

26. El-Sherbiny IM, Salama A and Sarhan AA: Ionotropically cross-linked $\mathrm{pH}$-sensitive IPN hydrogel matrices as potential carriers for intestine-specific oral delivery of protein drugs. Drug Dev Ind Pharm 37: 121-130, 2011.

27. Xiong W, Gao X, Zhao Y, Xu H and Yang X: The dual temperature/pH-sensitive multiphase behavior of poly ( $\mathrm{N}$-isopropylacrylamide-co-acrylic acid) microgels for potential application in in situ gelling system. Colloids Surf B Biointerfaces 84: 103-110, 2011.

28. Yin YH, Yang YJ and Xu BH: Swelling kinetics of hydrogels for colonic-site drug delivery. Acta Polymerica Sinica 2001: 650-655, 2001 (In Chinese).

29. Yin YH, Yang YJ and $\mathrm{Xu}$ BH: Hydrogels containing 4,4'-di(methacryloylmino)azobenzenes: Synthesis and animal experiment for colonic-site drug release. Acta Polymerica Sinica 2002: 408-413, 2002 (In Chinese).
30. Kim S, Kim JH and Kim D: pH sensitive swelling and releasing behavior of nano-gels based on polyaspartamide graft copolymers. J Colloid Interface Sci 356: 100-106, 2011.

31. Kono K, Tabata $\mathrm{F}$ and Takagishi T: $\mathrm{pH}$-responsive permeability of poly (acrylic acid)-poly (ethylenimine) complex capsule membrane. J Memb Sci 76: 233-243, 1993.

32. Li XQ, Dong L, Liu ZH and Luo JY: Expression of gamma-aminobutyric acid A receptor subunits alpha1, beta1, gamma2 mRNA in rats with hepatic encephalopathy. World J Gastroenterol 11: 3319-3322, 2005.

33. Zimmermann C, Ferenci P, Pifl C, Yurdaydin C, Ebner J, et al: Hepatic encephalopathy in thioacetamide-induced acute liver failure in rats: characterization of an improved model and study of amino acid-ergic neurotransmission. Hepatology 9: 594-601, 1989.

34. Chu CJ,Lee FY, Wang SS, Chang FY, Lin HC, et al: Establishment of an animal model of hepatic encephalopathy. Zhonghua Yi Xue Za Zhi (Taipei) 63: 263-269, 2000.

35. Yurdaydin C, Walsh TJ, Engler HD, Ha JH, Li Y, et al: Gut bacteria provide precursors of benzodiazepine receptor ligands in a rat model of hepatic encephalopathy. Brain Res 679: 42-48, 1995.

36. Bongaerts G, Severijnen R and Timmerman H: Effect of antibiotics, prebiotics and probiotics in treatment for hepatic encephalopathy. Med Hypotheses 64: 64-68, 2005.

37. Häberle J: Clinical practice: the management of hyperammonemia. Eur J Pediatr 170: 21-34, 2011.

38. Kowdley KV and Burman BE: ACP Journal Club. Adding rifaximin to lactulose increased reversal and decreased mortality in hepatic encephalopathy. Ann Intern Med 159: JC8, 2013.

39. Sharma BC, Sharma P, Lunia MK, et al: A randomized, double-blind, controlled trial comparing rifaximin plus lactulose with lactulose alone in treatment of overt hepatic encephalopathy. Am J Gastroenterol 108: 1458-1463, 2013.

40. Debray D, Yousef N and Durand P: New management options for end-stage chronic liver disease and acute liver failure: potential for pediatric patients. Paediatr Drugs 8: 1-13, 2006.

41. Sharma P, Sharma BC and Sarin SK: Predictors of nonresponse to lactulose for minimal hepatic encephalopathy in patients with cirrhosis. Liver Int 29: 1365-1371, 2009.

42. Maclayton DO and Eaton-Maxwell A: Rifaximin for treatment of hepatic encephalopathy. Ann Pharmacother 43: 77-84, 2009. 